\title{
Proceedings
}

\section{Breath Sounds as a Biomarker for Screening Infectious Lung Diseases ${ }^{\dagger}$}

Harini S. 1 , Parijat Deshpande ${ }^{2}$ and Beena Rai ${ }^{2, *}$

1 CTO, TCS Research, Pune, India; harini.s8@tcs.com

2 CTO, TCS Research, Pune, India; parijat.deshpande@tcs.com

* Correspondence: beena.rai@tcs.com

+ Presented at the 7th International Electronic Conference on Sensors and Applications, 15-30 November 2020; Available online: https://ecsa-7.sciforum.net/.

Published: 15 November 2020

\begin{abstract}
Periodic monitoring of breath sounds is essential for early screening of obstructive upper respiratory tract infections, such as inflammation of the airway typically caused due to viruses. As an immediate first step, there is a need to detect abnormalities in breath sounds. The adult average male lung capacity is approximately 6 litres and the manifestation of pulmonary diseases, unfortunately, remains undetected until their advanced stages when the disease manifests into severe conditions. Additionally, such rapidly progressing conditions arising due to viral infections that need to be detected via the adventitious breath sounds to take immediate therapeutic action demand frequent monitoring. These tests are usually conducted by a trained physician by means of a stethoscope, which requires an in-person visit to the hospital. During a pandemic situation like COVID-19, it is difficult for periodic screening of large volumes of people with the existing medical infrastructure. Fortunately, smartphones are ubiquitous, and even developing countries with skewed doctor-to-patient ratios typically have a smartphone in every household. With this technology accessibility in mind, we present a smartphone-based solution that monitors breath sounds from the user via the in-built microphone of their smartphone and our AI based anomaly detection engine. The presented automated classifier for abnormal breathing sounds is able to detect abnormalities in their early stages of respiratory dysfunctions w.r.t their individual normal baseline vesicular breath sounds with an accuracy of $95 \%$ and able to flag them thus enhancing the possibility of early detection.
\end{abstract}

Keywords: breath sounds; anomaly detection; feature extraction; classification; artificial neural networks; machine learning; convolution neural networks

\section{Introduction}

In humans, the respiratory system consists of the respiratory muscles, the lungs, and the airways. Breath sounds are the sounds produced by the structures of the lungs during breathing. The exchange of air between the atmosphere and the lungs (inspiration) and vice-versa (expiration) generates these breathing sounds. Breathing sounds are broadly classified as normal and abnormal breathing sounds. Normal breathing sounds can be loud, high pitched sounds heard over the trachea, medium pitched bronchovesicular sounds heard over the mainstream bronchi and soft, low pitched vesicular sounds heard over the chest. The most common types of abnormal breathing sounds are crackles, wheezes, rhonchi, and stridor [1]. Each of these abnormal breathing sounds can be a symptom of some serious respiratory diseases. So, listening to breath sounds is an important part of early diagnosis. In this paper we present an automated detection of anomalies in breath sounds using a smartphone [2-3] with nearly $95 \%$ accuracy against the user's normal baseline and offers a 
personalized report and trend of the results. In [1], measuring lung function is done by calculating the exhaled flow rate from the smartphone microphone. In [4], sequence classification models like HMM, LSTM variations are used to identify breath cycles followed by feedback. In [5], respiratory sounds are classified using adaptive neuro-fuzzy inference systems and Artificial Neural Networks using PSD as a feature. In [6], automation of lung analysis is carried out using time and frequency domain features like variance, sum of moving average, spectrum mean, etc. In [7], MFCC features are analyzed using one-way ANOVA and are fed to some classification algorithms. In [8], spectral and wavelet features are used with various classification algorithms. In [9], Mel frequency analysis of breathing sound is done with pattern recognition techniques to separate the breathing phases, to estimate the lung volume, and detect the presence of COVID-19.

In this paper, we have made use of sound sensor in the smartphone without requiring any external sensors. The sounds acquired are analyzed using various machine learning as well as deep learning algorithms.

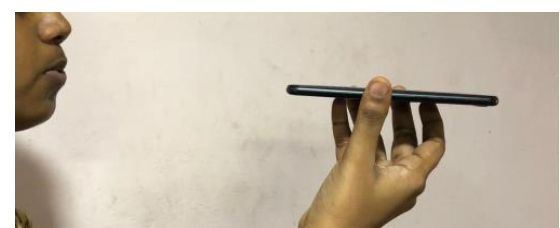

Figure 1. Shows a picture of a person recording her breath sounds by breathing into the smartphone's microphone.

\section{Methodology}

The flowchart of the steps used in this paper is represented in Figure 2.

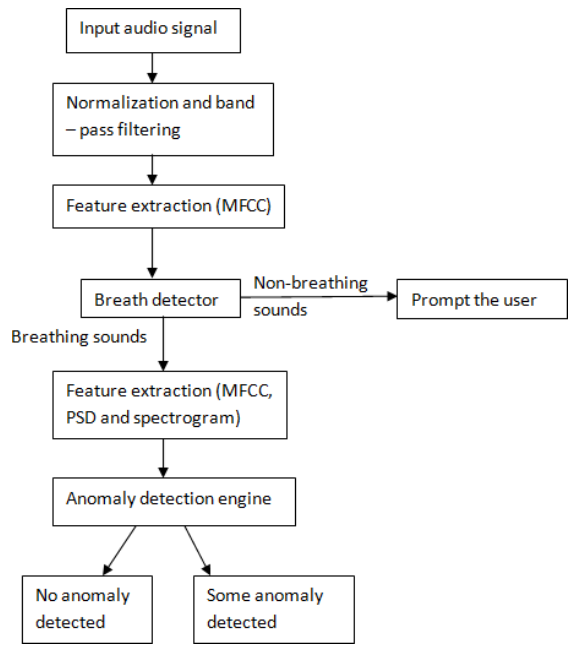

Figure 2. Flowchart of the steps used in this paper.

\subsection{Breath Detector}

The data used for breath detector is collected from various sources, including ESC-50 dataset [10]. The ESC-50 dataset is a publicly available dataset that provides a huge collection of speech and environmental sounds. This collection of sounds is categorized into 50 classes, one of them being breath sounds. We have used 592 breath sounds and 592 other environmental sounds for training our 
breath detector. As a pre-processing step, normalization is done to the signals in order to remove sharp or sudden change. Further, to remove background noise, the signals are filtered using a bandpass filter with a frequency range of 100-2500 Hz. We have calculated the Mel Frequency Cepstral Coefficients (MFCC) for audio samples. For the feature vector, we take the mean of MFCC features corresponding to all the frames [6], the feature vector is fed as input to the Breath detector which uses 3 different machine learning algorithms, which are Random Forest (RF), Logistic Regression (LR) and K-Nearest Neighbours (KNN) for binary classification.

We selected the final class label by majority voting on the predictions of all the 3 models.

\subsection{Anomaly Detection Engine}

The diagnosis engine gives the final result to the users whether they have normal or labored breathing. The data for normal and abnormal breathing sounds are collected from various data sources e.g., RALE database [11]. At the time of writing the paper, we had 295 normal and 295 abnormal sounds.

\subsubsection{Machine Learning Based Classifiers}

The audio samples are pre-processed and by applying Fast Fourier Transform (FFT), we calculated the Power Spectrum Density (PSD) of the data set. Since, PSD has excessive data points, we have decided to divide the PSD of each signal into 32 segments for which the individual averaged PSD is calculated. These 32 averaged values along with MFCCs make up the feature vector [5]. The feature vector is fed to 5 different learning algorithms [12], which are Artificial Neural Networks (ANN), Support Vector Machine (SVM), Random Forest (RF), Logistic Regression (LR) and KNearest Neighbours (KNN) for classifying into normal and labored breathing sounds [7]. The final class label is obtained by majority voting.

\subsubsection{Convolutional Neural Network}

The audio signals are pre-processed and spectrogram images are generated using FFT $([8,12])$. The images are resized into $28 \times 28$ grayscale image. The images are passed into $\mathrm{CNN}$ for classification $([13,14])$. The images are passed to two convolutional layers consisting of 32 filters and $3 \times 3$ kernel size with a $2 \times 2$ max-pooling layer in between to learn the complex features, which are then passed to another $2 \times 2$ max-pooling to represent the learned features in lower dimensions. The image is flattened to 1 dimension and then passed to a fully connected layer with 128 neurons. The final layer is the softmax classification layer with 2 neurons to distinguish between normal and labored sounds. ReLU activation function is used and Adam is used as optimizer.

\subsubsection{Ensembled Convolutional Neural Network}

We have used a form of ensembling which involves taking an average of outputs of models in the ensemble [15]. We have created two small CNNs and each model is trained separately on the training set of images and each isevaluated using the test set. First CNN model is a simple and similar to the one used in the above method. Second CNN model is a little more complex compared to the first. In this, we have used two sets of three convolution layers with $2 \times 2$ kernel size and filters of 32 and 64 respectively, which are followed by a max-pooling layer. Then, the images are passed to a convolution layer which generates 2 feature maps followed by global average pooling layer and a softmax classification. Further we have put the two models in an ensemble for evaluation. The results depict that the ensemble performs better on a test set than a single model.

\subsubsection{Gated-Convolutional Recurrent Neural Network}

The inspiration of this model comes from the work by [16]. The Gated-CRNN model has two convolutional layers followed by a Gated Recurrent Unit (GRU). The models use a dropout of 0.1 and 0.3 respectively and finally followed by a sigmoid layer. This model requires lesser memory usage 
and quite faster than the other models, since GRU has relatively lesser parameters. Categorical cross entropy is used as a loss function for this model.

\section{Results}

To evaluate the models, we have used macro average of the performance metrics of accuracy, sensitivity/recall, precision, F1-score on both the classes. These performance metrics are based on confusion matrices.

\subsection{Breath Detector}

The performance metrics of all the models we have used for breath detection are reported in Table 1.

Table 1. Performance metrics for breath detector.

\begin{tabular}{|c|c|c|c|}
\hline \multicolumn{4}{|c|}{ ModelTest Accuracy (\%)Precision (\%)Recall (\%) } \\
\hline KNN & 99.39 & 98.00 & 99.00 \\
\hline RF & 99.10 & 98.00 & 98.00 \\
\hline LR & 98.79 & 98.00 & 98.00 \\
\hline
\end{tabular}

3.2. Anomaly Detection Engine

The performance metrics for machine learning based classifiers in the anomaly detection engine are reported in Table 2 .

Table 2. Performance metrics for machine learning based classifiers in the anomaly detection engine.

\begin{tabular}{cccc}
\hline Model & Test Accuracy $\mathbf{( \% )}$ & Precision (\%) & Recall (\%) \\
\hline LR & 91.35 & 91.00 & 91.00 \\
SVM & 93.60 & 93.00 & 94.00 \\
ANN & 94.70 & 92.00 & 90.00 \\
RF & 91.01 & 92.00 & 90.00 \\
KNN & 91.50 & 92.00 & 90.00 \\
\hline
\end{tabular}

The confusion matrix for the CNN model, ensemble CNN model and Gated-RCNN is reported in Figure 3. At the moment, with limited data available, the overall accuracy of deep learning based binary-class classifier is $94 \%$. Future work will continue to improve this model as more training data becomes available for CNN.

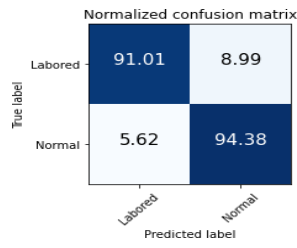

(a)

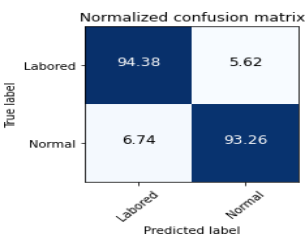

(b)

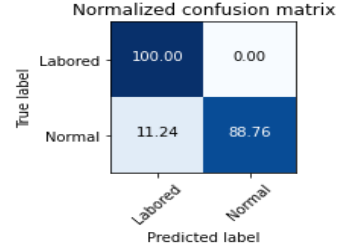

(c)

Figure 3. Normalized confusion matrix for (a) CNN model; (b) ensemble CNN model; and (c) GatedRCNN model (in percentage).

\section{Discussions}

The presented solution can further be extended into a platform to aid screening of various lung diseases on the basis of breath sounds. A cloud based implementation will allow use of AI algorithms 
that necessitate greater computation. Additional patient data will further improve accuracy and specific detection.

\section{Patents}

Detecting, M.F.O.R. Systems and Methods for Detecting Pulmonary Abnormalities Using Lung. 2019, 1 .

Deshpande, P; Vempada, R.; Dasgupta, R. Pal, A; Roy, D; United States Patent. 2018.

\section{Conclusions}

In this paper we present an AI based tool for the detection of anomalies in breath sounds acquired via a smartphone with an accuracy of $95 \%$ via machine learning and deep learning techniques. This application can serve as an aid and an essential tool for early detection of anomalies in breath sounds such as experienced in the recent pandemic situation caused due to COVID-19.

Supplementary Materials: A short video of this work is available online at https://healthsounds.cl.cam.ac.uk/workshop20/shorts/harini.mp4.

Acknowledgments: This work is done as a part of internship project at Tata Research and Development and Design Centre (TRDDC), Pune, India.

\section{References}

1. Lung, J. Breath Sounds Study Guide: The Ultimate Guide to Lung sounds and Auscultation; 2020

2. Larson, E.C.; Goel, M.; Boriello, G.; Heltshe, S.; Rosenfeld, M.; Patel, S.N. SpiroSmart 2012, 280, doi:10.1145/2370216.2370261.

3. Cheng, Q.; Juen, J.; Bellam, S.; Fulara, N.; Close, D.; Silverstein, J.C.; Schatz, B. Predicting Pulmonary Function from Phone Sensors. Telemed. E Health 2017, 23, 913-919, doi:10.1089/tmj.2017.0008.

4. Shih, C.-H.; Tomita, N.; Lukic, Y.; Reguera, Á.H.; Fleisch, E.; Kowatsch, T. Breeze: Smartphone-based Acoustic Real-time Detection of Breathing Phases for a Gamified Biofeedback Breathing Training. Proc. ACM Int. Mobile Wearable Ubiquitous Technol.2019, 3, 1-30, doi:10.1145/3369835.

5. Oweis, R.J.; Abdulhay, E.W.; Khayal, A.; Awad, A. An alternative respiratory sounds classification system utilizing artificial neural networks. Biomed. J. 2015, 38, 153-161, doi:10.4103/2319-4170.137773.

6. Morten, G. Automated Lung Sound Analysis. Arctic Univ. Norw. 2016, 16, 18.

7. Palaniappan, R.; Sundaraj, K.; Ahamed, N.U. Machine learning in lung sound analysis: A systematic review. Biocybern. Biomed. Eng. 2013, 33, 129-135, doi:10.1016/j.bbe.2013.07.001.

8. Datta, S.; Dutta Choudhury, A.; Deshpande, P.; Bhattacharya, S.; Pal, A. Automated lung sound analysis for detecting pulmonary abnormalities. Proc. Ann. Int. Conf. IEEE Eng. Med. Biol. Soc. EMBS 2017, 4594 4598, doi:10.1109/EMBC.2017.8037879.

9. Faezipour, M.; Abuzneid, A. Smartphone-Based Self-Testing of COVID-19 Using Breathing Sounds. Telemed. E Health 2020, doi:10.1089/tmj.2020.0114.

10. Piczak, K.J. ESC: Dataset for environmental sound classification. In Proceedings of the 2015 ACM Multimedia Conference, Brisbane, Australia, 26-30 October 2015; pp. 1015-1018, doi:10.1145/2733373.2806390.

11. RALE: A computer-assisted instructional package. Respir Care 1990, 35, 1006.

12. Palaniappan, R.; Sundaraj, K.; Sundaraj, S. A comparative study of the svm and k-nn machine learning algorithms for the diagnosis of respiratory pathologies using pulmonary acoustic signals. BMC Bioinform. 2014, 15, 1-8, doi:10.1186/1471-2105-15-223.

13. Riella, R.J.; Nohama, P.; Maia, J.M. Departamento Method for automatic detection of wheezing in lung sounds. J. Med. Syst. 2012, 36, 1707-1717, doi:10.1007/s10916-010-9631-8.

14. Aykanat, M.; Kılıç, Ö.; Kurt, B.; Saryal, S. Classification of lung sounds using convolutional neural networks. Eur. J. Image Video Proc. 2017, 2017, doi:10.1186/s13640-017-0213-2.

15. Saraiva, A.A.; Santos, D.B.S.; Francisco, A.A.; Vigno, J.; Sousa, M. Classification of Respiratory Sounds with Convolutional Neural Network; 2019.

Commented [m1]: Please add the publisher and location.

Commented [m2]: Please add the article title or journal name.

Commented [m3]: Newly added information, please confirm.

Commented [m4]: Please add the author name.

Commented [m5]: Please add the publisher and location. 
16. Viswanath, V.; Garrison, J.; Patel, S. SpiroConfidence: Determining the Validity of Smartphone Based Spirometry Using Machine Learning. In Proceedings of the 2018 40th Annual International Conference of the IEEE Engineering in Medicine and Biology Society (EMBC), Honolulu, HI, 17-21 July 2018; pp. 54995502, doi:10.1109/EMBC.2018.8513516.

Publisher's Note: MDPI stays neutral with regard to jurisdictional claims in published maps and institutional affiliations. (http://creativecommons.org/licenses/by/4.0/). 